\title{
3D RECONSTRUCTION OF ICE SHAPE USING VISIBLE AND THERMAL RANGE IMAGING FOR AIRCRAFT ICING STUDY
}

\author{
V. A. Knyaz ${ }^{1,2}$, E.V. Ippolitov ${ }^{3}$, M.M. Novikov ${ }^{3}$ \\ ${ }^{1}$ State Research Institute of Aviation System (GosNIIAS), 125319 Moscow, Russia - knyaz@gosniias.ru \\ ${ }^{2}$ Moscow Institute of Physics and Technology (MIPT), Dolgoprudny, Russia \\ ${ }^{3}$ Institute on Laser and Information Technologies of RAS - \\ branch Research Centre Crystallography and Photonics RAS, Shatura, Russia - ippevg@yandex.ru, novikov@ rambler.ru
}

Technical Commission II, WG 10

KEY WORDS: aircraft icing, 3D reconstruction, calibration, thermal imaging, ice wind tunnel, structured light, stereolithography

\begin{abstract}
:
Aircraft icing is one of the main factors decreasing the flight safety. Qualitative and quantitative understanding of the icing process is crucially needed for developing anti-icing measures and safety recommendations. Changes in aerodynamic characteristics of aircraft caused by changes in shape of aircraft surfaces due to the ice accretion can lead to significant aerodynamic performance degradation. So the reliable and accurate information of how the shape of the ice accretion influences on aerodynamic characteristics is a key point for predicting the changes in aerodynamic performance.

The study addresses to a problem of accurate shape measuring of ice accretion for further experimental study of iced-aircraft aerodynamic in a hydrodynamic tunnel. For this purpose the evaluation of various techniques of ice 3D measurements is performed that include as visible so thermal imaging of ice accretion. The results of evaluation serves for the decision of preferable technique to be used in experimental study. Also the framework is developed for creating physical models of iced aircraft based on result of real ice accretion shape measurement. It allows to produce stereolithography (SLA) copies of of an aircraft under icing condition for different levels of icing. These SLA-models of an aircraft under icing condition are then used for flow behaviour study in order to identify critical flying condition.
\end{abstract}

\section{INTRODUCTION}

The study of aircraft icing process is an essential part in providing the safety of flights. Such study includes two parts: (1) the study of aircraft icing process that gives information of how icing is developing in time under the given conditions and (2) the study of how icing of various shape and degree influence on aerodynamic characteristics of a plane.

Due to extreme difficulty of studying icing process in real aircraft flight, most of the experiments is carried out in aerodynamic or hydrodynamic tunnels. They allow to model given flight conditions and to monitor and control the flow process. The adequacy of the results obtained is based on a similitude concept saying that a model correctly reflects real application if geometric, kinematic and dynamic similarity is observed.

The first part of aircraft icing study is usually performed in icing wind tunnels (Lee et al., 2014), that provide controllable conditions to study ice accretion developing. At this stage ice shape and environment parameters are registered.

At the second stage the registered ice accretion shapes are used for numerical simulation of flight or for physical experiments in aerodynamic or hydrodynamic tunnels. These experiments allow to identify critical conditions for flight safety causing by icing.

This research is a part of a long-term research project aimed at exploring an influence of aircraft icing on flying characteristics

\footnotetext{
${ }^{*}$ Corresponding author
}

of aircraft by flow modeling in low-speed hydrodynamic tunnel. At this stage of the project different techniques for ice shape 3D reconstruction are evaluated to chose the most appropriate one for the considered task.

The paper presents the results of the experimental study of different technique for ice shape 3D reconstruction and the developed framework for registering icing process and re-producing it in stereolithography models for studying changes in aircraft aerodynamics by experiments in hydrodynamic tunnel.

The main contributions of this paper are (1) experimental evaluation of applicability thermal imaging for measuring 3D shape of ice; (2) comparative study of accuracy of ice shape reconstruction for different types of illumination condition; (3) a pipeline for iced aircraft model producing for aerodynamic study in hydrodynamic tunnel.

\section{RELATED WORK}

The problems of aircraft icing attract a lot of scholar attention due to the importance of timely identification of the icing process and understanding of its influence on aerodynamic performance. The area of the study includes the analysis of the state of the air that causes icing (Cao et al., 2018), in-flight icing detection (Ikiades et al., 2007), measuring the shape of ice accretion and its impact on aerodynamic characteristics.

The review (Cao et al., 2018) provides the systematic analysis of the recent researches on aircraft icing. The study considers the 
causes of icing and their relation with environmental and aircraftrelated parameters, types and severity of aircraft icing, and icing impact on aircraft performance. The analysis and summary of influence of different effects of ice accretion on aircraft flying characteristics are given, along with the survey of modern techniques for ice accretion measuring and predicting. Also, the review of aircraft flight accidents provoked by icing is performed.

In-time ice accretion detection on the surfaces of aircraft is crucially important for flight safety. Traditional techniques for ice detection measure the temperature of ambient air and the quantity of liquid water in the air to make decision of icing condition. The approach (Ikiades et al., 2007) proposes to analyze an optical diffusion of light in the ice volume for detecting ice and determine its type. The performed experiments in measuring light diffusion have shown that the type of the ice can be recognized as a result of analysis of spatial distribution of the specular and scattered components of light. Also the proposed technique can be exploited for estimating the thickness of the ice accretion.

But not only the thickness, but also mainly the shape of the ice accretion significantly affects the aerodynamic characteristics of the aircraft. Ice shape measuring is necessary for aircraft icing studying as by experiments in aerodynamic tunnels so by numerical simulation. Various techniques are used for ice 3D shape reconstruction. They can be divided into two groups: contact measurements and non-contact measurements.

For ice shape 3D reconstruction as contact so non-contact techniques are applied. Main disadvantage of contact methods (such as e.g., mold and casting) in measuring ice shape is interrupting the icing process, thus preventing its registration in time. Among the non-contact measurement techniques such as optical, ultrasonic (Hansman et al., 1988) and microwave, the former have a significant advantage in density and accuracy of measurements. The main problem for optical techniques in measuring ice accretion is reflective properties of ice surface.

A set of researches has been aimed to 3D measuring of ice shape for further experimental study of aerodynamics change in aerodynamic or hydrodynamic tunnels (Broeren et al., 2010), (Pouryoussefi et al., 2016), (Lee et al., 2019).

Ice-accretion models (casts) were used for evaluating the aerodynamic accuracy of ice accretion simulation (Lee et al., 2019). These model were produced from 3D surface models obtained by laser scanning technique. Experiments were performed in the NASA Icing Research Tunnel using an 18-inch chord, 2-D straight wing with NACA 23012 airfoil section. Ice accretion shape was acquired for six ice-accretion cases, which then have been used for creating hard copies of the accretion. The experiments were carried out with applying of special opaque spray to provide Lambertian reflectivity of ice surface.

This study is inspired by recent achievements in surface 3D reconstruction using thermal imaging (Knyaz et al., 2017), (Mizginov, Kniaz, 2019), (Gong, Bansmer, 2015), (Knyaz, Moshkantsev, 2019) and is aimed at comparative evaluating the applicability of thermal imaging for ice shape 3D reconstruction.

\section{PROJECT OUTLINE}

The current work is a part of the research project (Knyaz et al., 2020) aimed at experimental studying of aircraft icing. The main problems to be investigated are (1) accurate 3D measuring of flow in a hydrodynamic tunnel (HDT) for the impact of aircraft icing study; (2) aircraft icing process modelling for experiments in a low-speed hydrodynamic tunnel; (3) flow registration for iced surfaces condition and its accurate 3D reconstruction.

The project framework is shown in Figure 1.

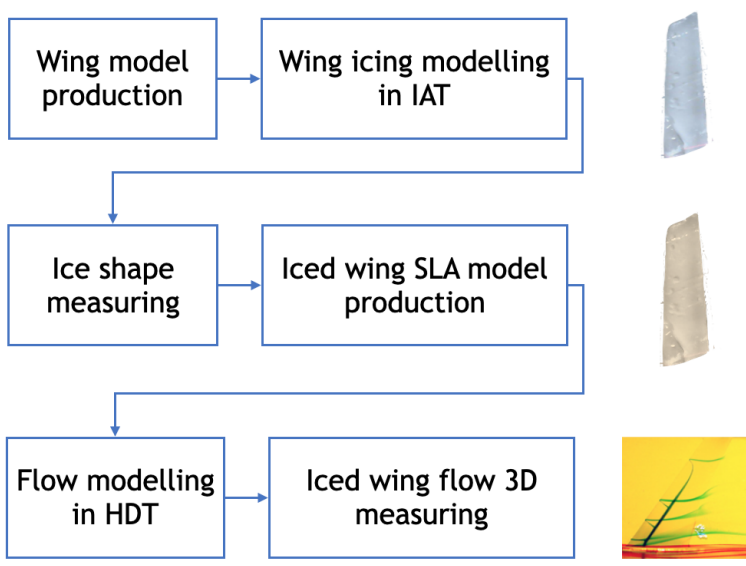

Figure 1. The project framework

Firstly, the icing process is studied in ice aerodynamic tunnel (IAT) using a model of an aircraft (or a part of an aircraft). The part model is produced by stereolithography (SLA) providing the required shape correspondence with the original. Then SLA model is set up in the ice aerodynamic tunnel, that provides icing conditions, resulting in ice accretion on the surface of the model.

The all-season artificial icing facility is a room in which an openloop fan-type wind tunnel, crystal injection and water spray systems are installed (Figure 2). The air temperature required for icing studies in the chamber reaches $-18^{\circ} \mathrm{C}$ and is set by injecting cold air into it, cooled in the refrigerator. The jet flowing out of the wind tunnel to improve its mixing with the surrounding air is broken up with the help of grids and fairings.

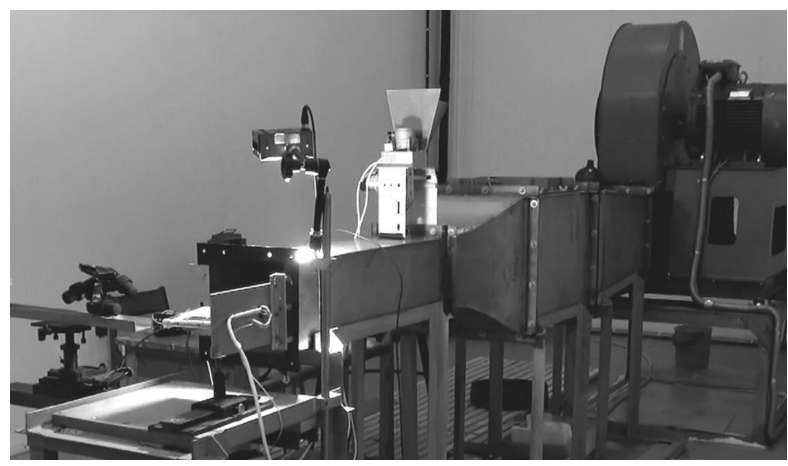

Figure 2. Icing wind tunnel

The stand provides a study of icing processes in the laboratory, regardless of the weather. The volume of the room of $360 \mathrm{~m}^{3}$ ensures the operation of a wind tunnel with an outlet section of the nozzle of $250 \times 250 \mathrm{~mm}^{2}$ at a flow rate of up to $80 \mathrm{~m} / \mathrm{s}$.

At the second step, the shape of the ice accretion is to 3D reconstruct for further reproducing at the experiments in a hydrodynamic tunnel. The result of the ice accretion $3 \mathrm{D}$ scanning is the 
3D model of the part with ice accretion (and its hard SLA model), that allows to model flow behaviour on iced surface. The current work is addressed to developing optical 3D measurement techniques for accurate $3 \mathrm{D}$ reconstruction of the ice accretion shape.

Finally, experiments are performed with the SLA model of an iced surface in the low-speed hydrodynamic tunnel, that serve for registering and $3 \mathrm{D}$ measuring of the flow disturbed by ice accretion.

\section{EXPERIMENTAL SETUP}

To provide comparable results of various 3D scanning techniques for all imaging/lighting configurations laser triangulation technique was chosen as $3 \mathrm{D}$ reconstruction method. The working space of $3 \mathrm{D}$ measurements was $300 \times 300 \times 300 \mathrm{~mm}$.

For ice accretion shape 3D reconstruction several configurations of photogrammetric 3D scanning system were used, based as on visible range camera so on thermal range camera.

\subsection{Thermal range configuration}

In thermal range configuration MH SM567 thermal camera serves for image acquisition and Iradion ${ }^{\circledR}$ Infinity $\mathrm{CO}_{2}$ laser was used for object lighting. Main specifications of the thermal camera and long-wave infra red laser are presented in Table 1.
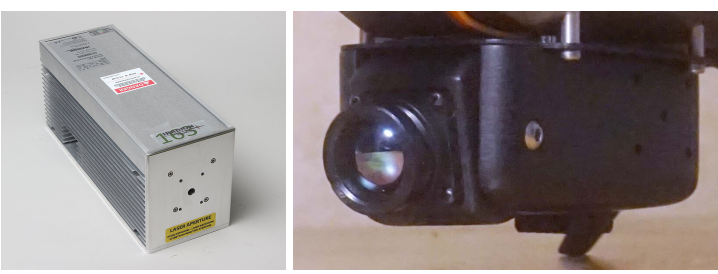

Figure 3. Iradion ${ }^{\circledR}$ Infinity $\mathrm{CO}_{2}$ laser and MH SM567 thermal camera

\begin{tabular}{lr}
\hline \hline Parameter & Value \\
\hline Wavelength, $\mu m$ & 9.3 \\
Rated Optical Power, $W$ & 25 \\
Beam ellipticity, $\mathrm{mm}$ & $<1.2: 1$ \\
Beam diameter, $\mathrm{mm}$ & $2.3 \pm 0.3$ \\
Beam divergence, mrad & $<7$ \\
Rise Time, $\mu$ sec & 1.3 \\
Power stability from cold, $\%$ & $\pm 7 \%$ \\
\hline \hline
\end{tabular}

Table 1. Main characteristics of Iradion ${ }^{\circledR}$ Infinity $\mathrm{CO}_{2}$ laser

Main technical characteristics of MH-SM567-6 are given in Table 2.

Table 2. Thermal and visible cameras specification

\begin{tabular}{lrr}
\hline \hline Parameter & MH-SM567 & DMK 37BUX273 \\
\hline Type & Uncooled FPA & $1 / 2.9 "$ CMOS \\
FPA format & $640 \times 480$ & $1,440 \times 1,080$ \\
Pixel pitch & $17 \mu \mathrm{m}$ & $4.45 \mu \mathrm{m}$ \\
Sensitivity & $\leq 150 \mathrm{mK}$ & \\
Frame rate & $50 \mathrm{~Hz}$ & up to 238 fps \\
Spectral range & $8-14 \mu \mathrm{m}$ & $0.4-0.95 \mu \mathrm{m}$ \\
Lens & $12.8 \mathrm{~mm}$ & $12 \mathrm{~mm}$ \\
\hline \hline
\end{tabular}

\subsection{Visible range configuration}

For 3D measuring in visible range DMK 37BUX273 USB 3.1 monochrome industrial camera was used with laser diode modules of different wave length. Main technical characteristics of DMK 37BUX273 camera are presented in Table 2, and specifications of laser diode modules are given in Table 3.

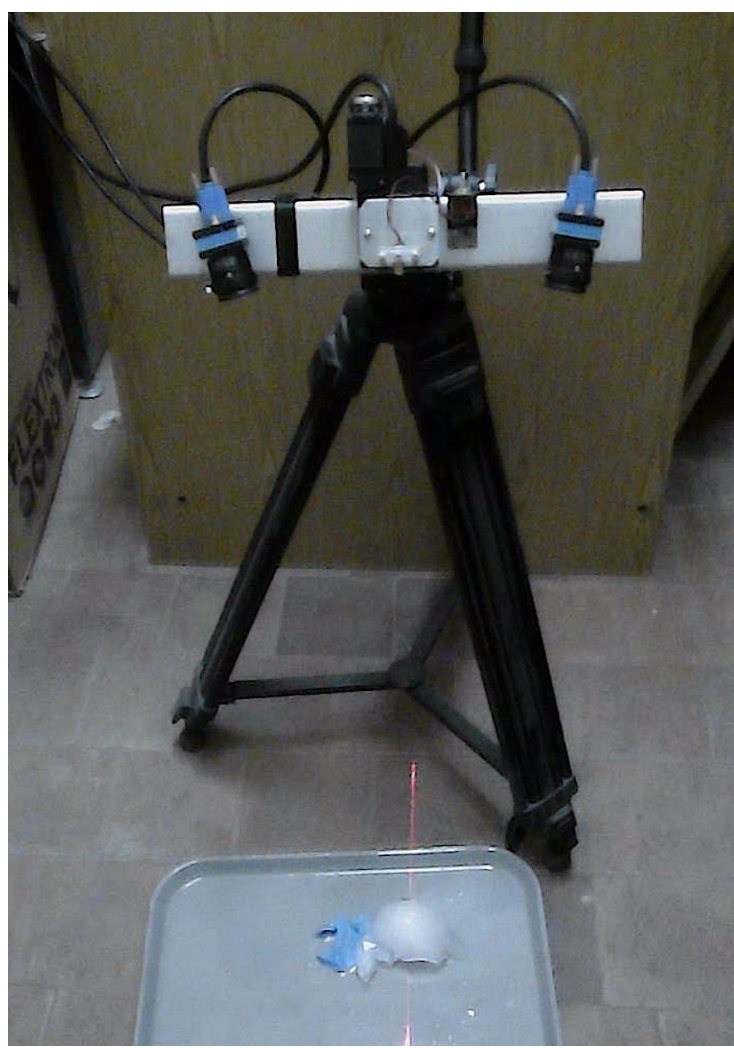

Figure 4. Visible range setup

\begin{tabular}{lrrr}
\hline \hline Parameter & Value & & \\
\hline Wavelength, $\mu m$ & 0.445 & 0.532 & 0.650 \\
Power, $m W$ & 1000 & 300 & 3 \\
Beam diameter, $m m$ & 2 & 3 & 3 \\
Beam divergence, mrad & $<2$ & $<2$ & $<0.6$ \\
Rise Time, $\mu$ sec & $<5$ & $<15$ & $<5$ \\
Power stability from cold, $\%$ & $\pm 5 \%$ & $\pm 5 \%$ & $\pm 5 \%$ \\
\hline \hline
\end{tabular}

Table 3. Main characteristics of laser diode modules

\subsection{Calibration}

The calibration of the $3 \mathrm{D}$ measuring system is prerequisite stage providing the required accuracy of the measurements. The basic imaging model for camera calibration is central projection, expressed in form of co-linearity equations describing the relation between the object point $S$, the corresponding image point $s$ and the center of projection $C$ :

$$
\mathbf{x}_{s}-\mathbf{x}_{p}=-\lambda \mathbf{R} \cdot\left(\mathbf{X}_{S}-\mathbf{X}_{C}\right)
$$

Here

$\mathbf{X}_{C}=\left(X_{C}, Y_{C}, Z_{C}\right)^{T}$ - coordinates of the projection center,

$\mathbf{X}_{S}=\left(X_{S}, Y_{S}, Z_{S}\right)^{T}$ - coordinates of the object point $S$, $\mathbf{x}_{s}=\left(x_{s}, y_{s},-c\right)^{T}-$ the corresponding image coordinates of the 
object point $S$,

$\mathbf{R}$ - rotation matrix of coordinate system transformation,

$\mathbf{x}_{p}$ - principal point coordinates

$\lambda$ - scale factor.

Two systems of coordinates are used to define the relation between the object point $S$ and its image $\mathbf{x}_{s}$ : (1) object coordinate system $O X Y Z$ that is related with the object and (2) image coordinate system $C x y z$ that is related with image plane. In Eq. 1 the object point $S$ and the projection center $C$ are determined in $O X Y Z$ system of coordinates, and the image point $\mathbf{x}_{s}-$ in $C x y z$ system of coordinates.

The transformation of digital (pixel) image coordinates $x_{s}^{d}, y_{s}^{d}$ of the point $S$ to its image coordinates $\mathbf{x}_{s}=\left(x_{s}, y_{s}, z_{s}\right)^{T}$ is defined by the following Eq. 2 - 4:

$$
\begin{aligned}
& x_{s}=m_{x} \cdot\left(x_{s}^{d}-x_{p}\right) \\
& y_{s}=m_{y} \cdot\left(y_{p}-y_{s}^{d}\right) \\
& z_{s}=-c .
\end{aligned}
$$

For accurate image-based 3D measurements the basic imaging model (1) should be modified for taking into account distortions introduced by real imaging system. The correction of the distorted image coordinates $(x, y)$ to obtain undistorted coordinates $(\tilde{x}, \tilde{y})$ can be written as:

$$
\begin{aligned}
& \tilde{x}=x+\Delta x ; \\
& \tilde{y}=y+\Delta y ;
\end{aligned}
$$

with $(\Delta x, \Delta y)$ being additional terms describing various distorting factors.

The Brown-Conrady distortion model (Brown, 1966) is widely used in photogrammetric applications, taking $(\Delta x, \Delta y)$ in the following form (Beyer, 1992)::

$$
\begin{aligned}
& \Delta x=a_{0} \cdot y+x\left(a_{1} r^{2}+a_{2} r^{4}+a_{3} r^{6}\right)+a_{4}\left(r^{2}+2 x^{2}\right)+2 a_{5} x y \\
& \Delta y=a_{0} \cdot x+y\left(a_{1} r^{2}+a_{2} r^{4}+a_{3} r^{6}\right)+a_{5}\left(r^{2}+2 y^{2}\right)+2 a_{4} x y
\end{aligned}
$$

where $r^{2}=x^{2}+y^{2}$, and

$x, y$ are image coordinates of a point,

$a_{0}, \ldots, a_{5}$ are the interior orientation parameters of a camera, with:

$a_{0}$ - coefficient of affine distortion;

$a_{1}, a_{2}, a_{3}$ - coefficients of radial distortion;

$a_{4}, a_{5}$ - coefficients of tangential distortion.

The parameters of the distortion model are determined as a result of calibration procedure, starting with acquiring a set of test field images with known coordinates given reference points. Then least mean square estimation is used for determining unknown parameters of the distortion model, considering image coordinates of test field reference points as the observations.

The vector of unknown parameters of the interior orientation $v_{e}^{l}=$ $\left(x_{p}, y_{p}, m_{x}, m_{y}, a_{0}, \ldots, a_{5}\right)^{T}$ consists of coordinates of principal point, image scales and additional parameters correspondingly, spatial coordinates of reference points being known by independent precise measurements.
The same flat test field with 49 reference points was used for both (visible/thermal) cameras calibration. Sample images of the test field in visible and thermal range acquired by evaluated cameras are shown in Figure 5.
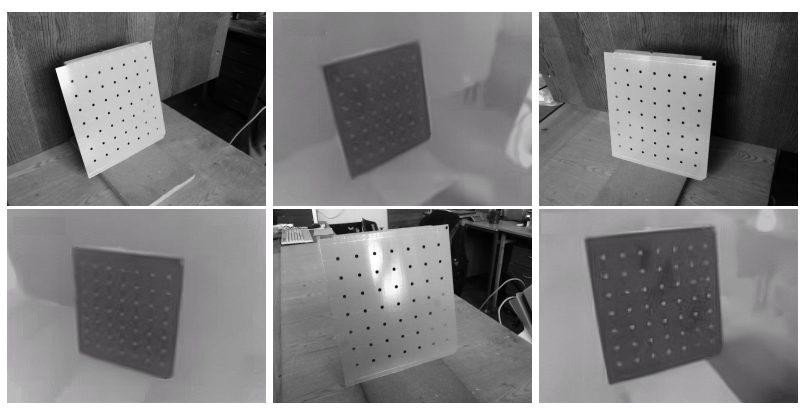

Figure 5. Samples of test field images in visible and thermal range used for calibration

Series of 15 images of the test field in different positions and orientations relatively to the camera were acquired by both visible and thermal range cameras. Automatically detected reference points in the images were considered as observations for original calibration procedure (Knyaz, 2006, Knyaz et al., 2021) for estimating parameters of the distortion model (Eq. 7 - 8).

The results of camera calibration for visible and thermal cameras are shown in Table 4.

\begin{tabular}{|cr|cr|}
\hline \multicolumn{2}{|c|}{$\begin{array}{c}\text { DMK 37BUX273 } \\
\text { (Visible range) }\end{array}$} & \multicolumn{2}{c|}{$\begin{array}{c}\text { MH-SM567 } \\
\text { (Thermal range) }\end{array}$} \\
\hline$m_{x}, m m$ & 0.0049273 & $m_{x}, m m$ & 0.01695168 \\
$m_{y}, m m$ & 0.0048791 & $m_{y}, m m$ & 0.01719864 \\
$x_{p}, p i x$ & 712.1072 & $x_{p}, p i x$ & 321.1843 \\
$y_{p}, p i x$ & 538.5521 & $y_{p}, p i x$ & 130.1494 \\
$a_{0}$ & -0.02277174 & $a_{0}$ & -0.01946129 \\
$a_{1}$ & 0.00150923 & $a_{1}$ & 0.00147901 \\
$a_{2}$ & 0.00000492 & $a_{2}$ & -0.00007257 \\
$a_{3}$ & -0.00000181 & $a_{3}$ & -0.00000170 \\
$a_{4}$ & -0.00189544 & $a_{4}$ & -0.00124871 \\
$a_{5}$ & -0.00051928 & $a_{5}$ & -0.00080137 \\
\hline
\end{tabular}

Table 4. Results of cameras calibration

\section{EVALUATION RESULTS}

First experiments were performed with ice structure to obtain qualitative estimate of possibilities to use structured lighting of different kinds. Figure 6 presents sample images of an ice object in visible range with laser module of red light $(650 \mu \mathrm{m})$ and the same ice object in thermal (long wave infra-red $8 \ldots 14 \mu \mathrm{m}$ ) range with structured light of $9.3 \mu \mathrm{m}$.

Figure 6(a) demonstrates that in case of image acquisition in visible range structured light does not provide appropriate structure on the ice surface, so shape 3D reconstruction is not possible by structured light technique. Special measures (like opaque spray) are needed to provide diffuse characteristics of the surface.

From the other hand thermal lighting is suitable for detecting structured light in the thermal image (Figure 6(b)), and consequently, to 3D reconstruct the shape of the ice structure without any additional measures. 


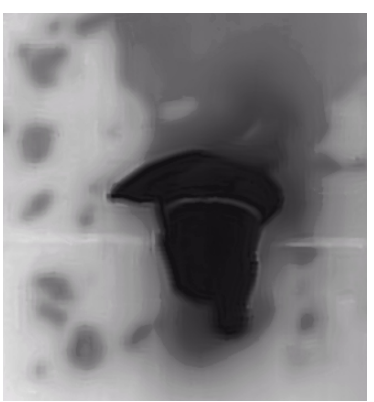

(a) ice structure vision range image with thermal $(9.3 \mu \mathrm{m})$ structured light

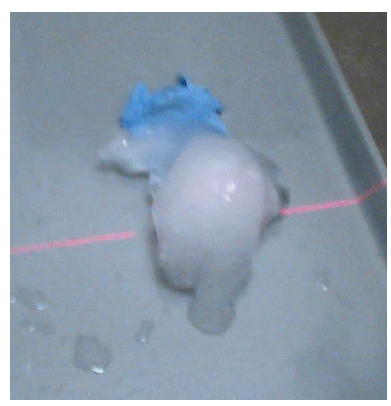

(b) ice structure visible image with red $(650 \mu \mathrm{m})$ structured light)

Figure 6. Ice structure images in visible and thermal range

At the second stage of the experiments, an ice accretion was formed on the surface of the wing model (Figure 7). This ice model was also scanned by vision and thermal system configurations.

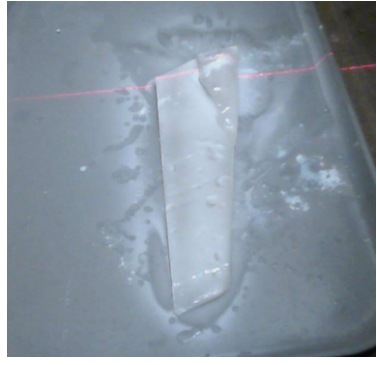

(a) ice accretion on a wing in vi sion image with red $(650 \mu \mathrm{m})$ structured light

Figure 7. Ice accretion on wing SLA model in visible and thermal range

Comparison of the obtained scans with the reference surface and one with other (Figures 8(a), 8(b)) has showed that in the case of a diffusely reflecting surface, the visible range configurations demonstrate better accuracy of $3 \mathrm{D}$ reconstruction. But the visible range configurations failed to correctly $3 \mathrm{D}$ reconstruct the clear icy surface.

From the other side, thermal system configuration demonstrated worse accuracy and resolution for diffusely reflecting surface, but does show capability 3D reconstruct ice surface with the quality reasonable for studying aircraft aerodynamics degradation due to icing (Figure 8(c)).

Preliminary experimental study has demonstrated that thermal 3D scanning can be applied for ice accretion 3D measurements, providing reasonable quality of the resulting $3 \mathrm{D}$ models. But the thermal 3D scanning configuration has notable drawbacks comparing with vision range configuration. These drawbacks are relatively high cost of the equipment and the complexity of maintenance.

Meanwhile the applying vision range configurations along with using opaque spray is more preferable for ice accretion $3 \mathrm{D}$ reconstruction at the first stage of the study.

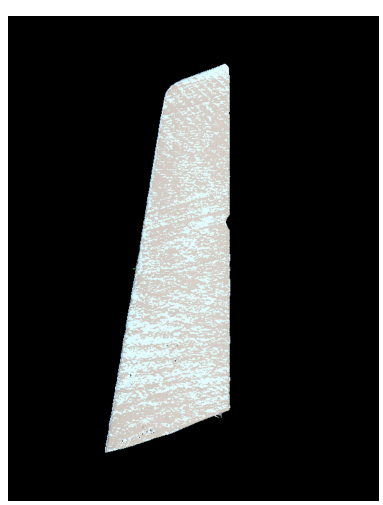

(a) comparison vision range $3 \mathrm{D}$ scan with thermal range 3D scan for clear wing

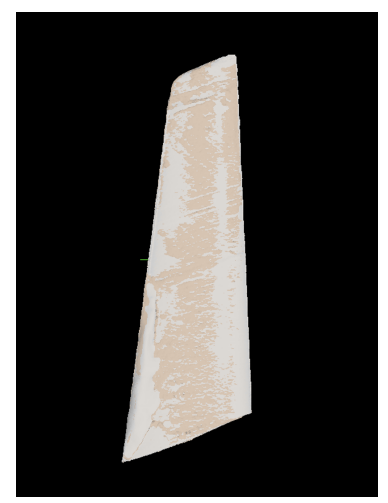

(b) comparison vision range $3 \mathrm{D}$ scan with thermal range 3D scan for icy wing

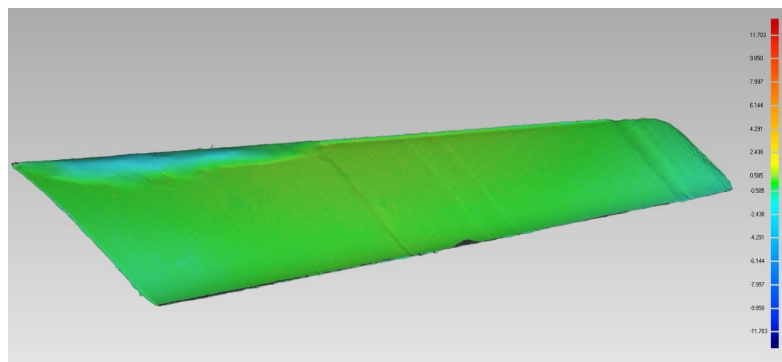

(c) comparison thermal range 3D scans for clear wing and icy wing

Figure 8. Results of 3D reconstruction and comparison of 3D models obtained in visible and thermal ranges

\section{CONCLUSION}

Experimental evaluation on test surfaces having diffuse reflectance has shown that optical 3D measurements in long-wave infra-red range have lower accuracy and resolution that visible range $3 \mathrm{D}$ scanning.

Meanwhile, thermal 3D scanning allows to reconstruct 3D shape of icy surfaces without applying the opaque spray, thus providing a possibility of $3 \mathrm{D}$ registering the process of ice accretion evolution in time on aircraft surfaces. Also thermal 3D scanning provides sufficient accuracy and resolution to be applied in aircraft icing study.

\section{ACKNOWLEDGEMENTS}

The reported study was funded by Russian Foundation for Basic Research (RFBR) according to the research project 19-29-13040.

\section{REFERENCES}

Beyer, H., 1992. Advances in Characterization and Calibration of Digital Imaging Systems. Int. Arch. Photogramm. Remote Sens. Spatial Inf. Sci., XXIX, 545-555.

Broeren, A. P., Whalen, E. A., Busch, G. T., Bragg, M. B., 2010. Aerodynamic Simulation of Runback Ice Accretion. Journal of Aircraft, 47(3), 924-939. https://doi.org/10.2514/1.46475.

Brown, D., 1966. Decentering Distortion of Lenses. Photogrammetric Engineering, 32(3), 444-462. 
Cao, Y., Tan, W., Wu, Z., 2018. Aircraft icing: An ongoing threat to aviation safety. Aerospace Science and Technology, 75, 353 - 385. http://www.sciencedirect.com/science/article/pii/ S1270963817317601.

Gong, X., Bansmer, S., 2015. 3-D ice shape measurements using mid-infrared laser scanning. Opt. Express, 23(4), 4908-4926. http://www.opticsexpress.org/abstract.cfm?URI=oe-23-4-4908.

Hansman, R. J., Kirby, M. S., McKnight, R. C., Humes, R. L., 1988. In-flight measurement of airfoil icing using an array of ultrasonic transducers. Journal of Aircraft, 25(6), 531-537. https://doi.org/10.2514/3.45618.

Ikiades, A., Howard, G., Armstrong, D. J., Konstantaki, M., Crossley, S., 2007. Measurement of optical diffusion properties of ice for direct detection ice accretion sensors. Sensors and Actuators A: Physical, 140(1), 24-31. https://www.sciencedirect.com/science/article/pii/ S0924424707004451.

Knyaz, V. A., 2006. Automated calibration technique for photogrammetric system based on a multimedia projector and a CCD camera. ISPRS - International Archives of the Photogrammetry, Remote Sensing and Spatial Information Sciences, XXXVI-5, 1-5. https://www.isprs.org/proceedings/XXXVI/part5/.

Knyaz, V. A., Moshkantsev, P. V., 2019. JOINT GEOMETRIC CALIBRATION OF COLOR AND THERMAL CAMERAS FOR SYNCHRONIZED MULTIMODAL DATASET CREATING. The International Archives of the Photogrammetry, Remote Sensing and Spatial Information Sciences, XLII2/W18, 79-84. https://www.int-arch-photogramm-remote-sensspatial-inf-sci.net/XLII-2-W18/79/2019/.

Knyaz, V. A., Stepaniants, D. G., Ippolitov, E. V., Novikov, M. M., 2020. OPTICAL 3D MEASUREMENTS IN HYDRODYNAMIC TUNNEL FOR AIRCRAFT ICING STUDY. The International Archives of the Photogrammetry, Remote Sensing and Spatial Information Sciences, XLIII-B2-2020, 963968. https://www.int-arch-photogramm-remote-sens-spatial-infsci.net/XLIII-B2-2020/963/2020/.

Knyaz, V. A., Stepaniants, D. G., Tsareva, O., 2021. Optical system calibration for 3D measurements in hydrodynamic tunnel. Computer Optics, 45(1), 58-65. http://computeroptics.ru.

Knyaz, V. A., Vygolov, O., Kniaz, V. V., Vizilter, Y., Gorbatsevich, V., Luhmann, T., Conen, N., 2017. Deep learning of convolutional auto-encoder for image matching and $3 \mathrm{~d}$ object reconstruction in the infrared range. 2017 IEEE International Conference on Computer Vision Workshops (ICCVW), 2155-2164.

Lee, S., Broeren, A., Kreeger, R., Potapczuk, M., Utt, L., 2014. Implementation and Validation of 3-D Ice Accretion Measurement Methodology. AIAA Paper, 2014-2613.

Lee, S., Broeren, A. P., Kreeger, R. E., Potapczuk, M. G., Utt, L., 2019. Implementation and Validation of 3-D Ice Accretion Measurement Methodology.

Mizginov, V. A., Kniaz, V. V., 2019. EVALUATING THE ACCURACY OF 3D OBJECT RECONSTRUCTION FROM THERMAL IMAGES. The International Archives of the Photogrammetry, Remote Sensing and Spatial Information Sciences, XLII-2/W18, 129-134. https://www.int-arch-photogrammremote-sens-spatial-inf-sci.net/XLII-2-W18/129/2019/.

Pouryoussefi, S. G., Mirzaei, M., Nazemi, M.-M., Fouladi, M., Doostmahmoudi, A., 2016. Experimental study of ice accretion effects on aerodynamic performance of an NACA 23012 airfoil. Chinese Journal of Aeronautics, 29(3), 585-595. https://www.sciencedirect.com/science/article/pii/ S100093611630019X. 